\title{
IR-Improved Results for LHC/FCC Physics: Status Report
}

\author{
B.F.L. Ward*广 \\ Baylor University, Waco, TX, USA \\ E-mail: BEL_Wardebaylor.edu \\ B. Shakerin \\ Baylor University, Waco, TX, USA \\ E-mail: Bahram_Shakerin@baylor.edu
}

\section{S.A. Yost}

The Citadel, Charleston, SC, USA

E-mail: Yost.S1@citadel.edu

We present the current status of recent developments in the theory and application of IR-improved $\mathrm{QED} \otimes \mathrm{QCD}$ resummation methods, realized by MC event generator methods, for LHC and FCC physics scenarios.

ICHEP2018, 39th International Conference on High Energy Physics

5-11 July 2018

Seoul, S. Korea

\footnotetext{
* Speaker.

${ }^{\dagger}$ On Research Leave from Baylor University, Waco, TX, USA, Jan. 4 - Jul. 31,2018, at Werner-Heisenberg-Institut, Max-Planck-Institut fuer Physik, Muenchen, Germany
} 
In the context of QCD, in addition to the original discussion ${ }^{1}$ of whether the naive JacksonScharre [1] or the exact YFS-style [2] resummation is more accurate for a given level of exactness, we have the issue of a hard cut-off for the IR versus resummed IR integrability. Ultimately, in the current era of QCD with precision tags $\lesssim 1.0 \%$ with accompanying EW precision tags at the per mille level for processes such as single heavy gauge boson production at the LHC, the precision data should be able to settle this additional issue as it resolves the original discussion for the QCD case.

In order to achieve enhanced precision for a given level of exactness while addressing the present paradigm in precision physics at the LHC and the futuristic FCC, we have pursued exact amplitude-based resummation realized on an event-by-event basis via shower/matrix element(ME) matched MC's. Currently, in the Herwig6.5 [3] environment we have a realization of IR-improved parton showers in the MC Herwiri1.031 [4] by two of us (BFLW and SAY). This MC is elevated to the exact NLO shower/ME matched level via the MC@NLO [5] and via the MG5_aMC@NLO [6] frameworks as MC@NLO/Herwiri1.031 [7] and MG5_aMC@NLO/Herwiri1.031 [8], respectively. We have the realization of IR-improved (IRI) Pythia8 [10] by one of us (BFLW) in the Pythia8 [9] environment, with its corresponding NLO shower/ME matched MG5_aMC@NLO/IRI-Pythia8. More recently, in the Herwig6.5 environment two of us (BFLW and SAY) have realized in the new MC $\mathscr{K} \mathscr{K}$ MC-hh [11] exact $\mathscr{O}\left(\alpha^{2} L\right)$ CEEX EW corrections in a hadronic MC.

Two of us (BFLW, SAY) have shown $[4,7]$ that IR-improvement in Herwig6.5 via Herwiri1.031 leads to improved precision in both the central $\left|\eta_{\ell}\right| \lesssim 2.5$ region for the ATLAS, CMS, D0 and CDF data and in the more forward region of LHCb where $2.0<\eta_{\ell}<4.5$. Here, $\left|\eta_{\ell}\right|$ is the lepton pseudorapidity in respective single $Z / \gamma^{*}$ production production with decay to lepton pairs. The availability of the IR-improved semi-analytical paradigm for the latter processes has been shown by one of us (BFLW) [12]. In what follows, we present a status report on our methods applied in the analysis of LHC W+ $\mathrm{n}$ jets data, to the FCC discovery physics and to the interplay of IR-improved parton showers with exact $\mathscr{O}\left(\alpha^{2} L\right)$ CEEX EW corrections in $\mathscr{K} \mathscr{K}$ MC-hh.

We note that 2017 was the 50th anniversary of the seminal paper by S. Weinberg [13] in which he formulated his foundational model of leptons in creating the spontaneously broken $S U_{2 L} \times U_{1}$ EW theory $[14,15]$, one of the key components of the SM, which we may now call the Standard Theory $(\mathrm{ST})^{2}$. Progress on precision theory has been essential to the establishment of the ST[13, $14,15,16,17]$. As we celebrate 50 years of the SM [18], we are also obliged to look to the future with the FCC [19] on the horizion, which will feature a $100 \mathrm{TeV}$ hadron collider and a tera- $\mathrm{Z} e^{+} e^{-}$ colliding beam device. The success of the latter devices will also depend strongly on the progress of precision theory.

The paper is organized as follows. After we briefly review the parton shower implementation of exact amplitude-based resummation theory, we turn to the interplay of IR-improved DGLAPCS QCD theory and shower/ME matched precision via comparisons with LHC data on $W+n$ jets and via predictions for FCC discovery physics. We finally discuss the interplay of IR-improved DGLAP-CS QCD theory and exact $\mathscr{O}\left(\alpha^{2} L\right)$ CEEX EW corrections in single $Z / \gamma^{*}$ production at the LHC.

\footnotetext{
${ }^{1}$ F. Berends, private communication, at 1988 ICHEP Conference Dinner, Munich, Germany.

${ }^{2}$ See D.J. Gross, talk, SM@50 Symposium, Cleveland, OH, June, 2018.
} 
The starting point for the parton shower implementation of exact amplitude-based resummation theory is the master formula

$$
\begin{gathered}
d \bar{\sigma}_{\mathrm{res}}=e^{\mathrm{SUM}_{\mathrm{IR}}(\mathrm{QCED})} \sum_{n, m=0}^{\infty} \frac{1}{n ! m !} \int \prod_{j_{1}=1}^{n} \frac{d^{3} k_{j_{1}}}{k_{j_{1}}} \\
\prod_{j_{2}=1}^{m} \frac{d^{3} k^{\prime} j_{2}}{k^{\prime} j_{2}} \int \frac{d^{4} y}{(2 \pi)^{4}} e^{i y \cdot\left(p_{1}+q_{1}-p_{2}-q_{2}-\sum k_{j_{1}}-\sum k^{\prime} j_{2}\right)+D_{\mathrm{QCED}}} \\
\tilde{\bar{\beta}}_{n, m}\left(k_{1}, \ldots, k_{n} ; k_{1}^{\prime}, \ldots, k_{m}^{\prime}\right) \frac{d^{3} p_{2}}{p_{2}^{0}} \frac{d^{3} q_{2}}{q_{2}^{0}}
\end{gathered}
$$

where new (YFS-style) non-Abelian residuals $\tilde{\bar{\beta}}_{n, m}\left(k_{1}, \ldots, k_{n} ; k_{1}^{\prime}, \ldots, k_{m}^{\prime}\right)$ have $n$ hard gluons and $m$ hard photons. The infrared functions $\operatorname{SUM}_{\mathrm{IR}}(\mathrm{QCED})$ and $D_{\mathrm{QCED}}$ and the residuals are defined in Ref. [7]. In the context of shower/ME matching, we have the replacements $\tilde{\bar{\beta}}_{n, m} \rightarrow \hat{\bar{\beta}}_{n, m}$ which allow us, via the basic formula

$$
d \sigma=\sum_{i, j} \int d x_{1} d x_{2} F_{i}\left(x_{1}\right) F_{j}\left(x_{2}\right) d \hat{\sigma}_{\text {res }}\left(x_{1} x_{2} s\right)
$$

to proceed with connection to MC@NLO as explained in Ref. [7].

Our (BS, BFLW) recent applications [20,21] make comparisons between the LHC data on $W+n$ jets, $\mathrm{n}=1,2,3$, and the exact NLO ME matched QCD parton shower predictions in the MG5_aMC@NLO framework with the parton shower realized via Herwig6.5 and Herwiri1.031 respectively for the unimproved and IR-improved results. We illustrate such results in Fig. 1 for the ATLAS $7 \mathrm{TeV}$ cms energy $W+1$ jet and

(a)

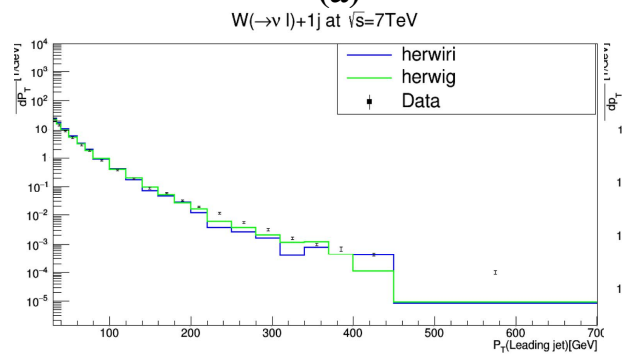

(b)

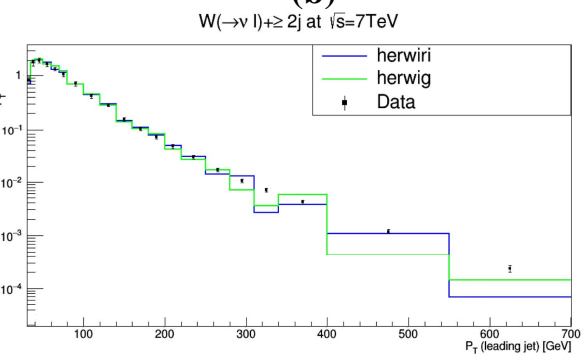

Figure 1: Comparison of ATLAS $7 \mathrm{TeV}$ cms energy $W+1$ jet (a) and $W+2$ jets (b) data for the leading jet $p_{T}$ distributions and the IR-improved(herwiri) and unimproved (herwig) exact NLO ME matched parton shower predictions.

$W+2$ jets data. Consistent with Refs. [7], the IR-improved results are closer to the data for low $p_{T}$.

The effect of IR-improvement on the discovery reach of a standard candle process such as single $Z / \gamma^{*}$ production at the FCC has been investigated by two of us (BFLW and SAY) using the predicted inclusive cross section for $Z / \gamma^{*}$ as a function of $p_{T, \min }{ }^{3}$ which is explained and shown in Fig. 6 in Ref. [22]. We plot in the latter figure predictions for the following: MG5_aMC@NLO/A, A= Herwig6.5, Herwiri1.031, Herwig++ and Pythia8, all with the common renormalization and factorization scale of $M_{Z} / 2$ and all with the common renormalization and factorization scale of $H_{T} / 2$ (denoted by 'UNFIX' in the legend in the figure); MG5_aMC@NLO/Herwig6.5 and fixed order NLO both with the common renormalization and factorization $M_{Z}$; and, fixed order NLO with the common renormalization and factorization scale $H_{T} / 2$. Here, $H_{T}$ is the sum of the transverse masses of the final state particles - see Ref. [22] for the remaining details. Our results show that the dynamical scale choice makes a big difference in the expectations, the fixed-order NLO results agree with the MG5_aMC@NLO/Herwig6.5 results for both of the scale choices, as it is expected, and the IR-improved and unimproved predictions agree within the statistical uncertainties.

Finally, in Refs. [23] two of us (BFLW and SAY) have analyzed the effects of the exact $\mathscr{O}\left(\alpha^{2} L\right)$ CEEX EW corrections in $\mathscr{K} \mathscr{K}$ MC-hh on the analysis of the $Z$ observables used in the ATLAS $M_{W}$ measurement in

\footnotetext{
${ }^{3}$ This observable was suggested by M.L. Mangano, private communication, 2016.
} 
Ref. [24]. We find new effects that should be considered at or above the per mille level. For example, the new modulation we see in the lepton $p_{T}$ spectrum seems to match the trends in the data vs theory comparisons shown in Figs. 15 a and b in Ref. [24]. We are extending the analysis in Ref. [23] to IR-improved showers with the new MC $\mathscr{K} \mathscr{K}$ MC-hh/Herwiri1.031 as explained and illustrated in Ref. [22]. One of us (BFLW) thanks Profs. S. Bethke and W. Hollik for the support and kind hospitality of Werner-Heisenberg-Institut, Max-Planck-Institut fuer Physik, Munich, Germany while part of this research was done.

\section{References}

[1] J.D. Jackson and D.L. Scharre, Nucl. Instum. Meth.128 (1975) 13.

[2] D. R. Yennie, S. C. Frautschi, and H. Suura, Ann. Phys. 13 (1961) 379; see also K. T. Mahanthappa, Phys. Rev. 126 (1962) 329, for a related analysis.

[3] G. Corcella et al., hep-ph/0210213; J. High Energy Phys. 0101 (2001) 010; G. Marchesini et al., Comput. Phys. Commun. 67 (1992) 465.

[4] S. Joseph et al., Phys. Rev. D81 (2010) 076008, arXiv:1001.1434.

[5] S. Frixione and B.Webber, J. High Energy Phys. 0206 (2002) 029; S. Frixione et al., arXiv:1010.0568; B. Webber, talk at CERN, 03/30/2011; S. Frixione, talk at CERN, 05/04/2011.

[6] A. Alwall et al., arXiv:1405.0301, and references therein.

[7] S.K. Majhi et al., Phys. Lett. B719 (2013) 367, arXiv:1208.4750; A. Mukhopadhyay and B.F.L. Ward, Mod. Phys. Lett. A 31 (2016) 1650063, arXiv:1412.8717.

[8] B. Shakerin and B.F.L. Ward, PoS(ICHEP2016) (2016) 1144; arXiv:1809.01492; BU-HEPP-17-05; BU-HEPP-18-02.

[9] T. Sjostrand, S. Mrenna and P. Skands, Comput. Phys. Commun. 178 (2008) 852; T. Sjostrand, arXiv:0809.0303, DOI:10.3204/DESY-PROC-2009-02/41; T. Sjostrand et al., Comput. Phys. Commun. 191 (2015) 159.

[10] B.F.L. Ward, Compt. Phys. Commun. 201 (2016) 29, arXiv:1504.00892.

[11] S. Jadach, B.F.L. Ward, Z. A. Was and S. A. Yost, Phys. Rev. D 94 (2016) 074006.

[12] B.F.L. Ward, Mod. Phys. Lett. A 31 (2016) 1650126, arXiv:1407.7290.

[13] Phys. Rev. Lett. 19 (1967) 1264.

[14] S. L. Glashow, Nucl. Phys. 22 (1961) 579.

[15] A. Salam, in Elementary Particle Theory, ed. N. Svartholm (Amquist and Wiksell, Stockholm, 1968).

[16] D.J. Gross and F. wilczek, Phys. Rev. Lett. 30 (1973) 1343.

[17] H.D. Ploitzer, Phys. Rev. Lett. 30 (1973) 1346.

[18] B.W. Lynn et al., to appear.

[19] M. Benedikt and F. Zimmermann, talk in 2017 FCC Week, Berlin, 2017.

[20] B. Shakerin and B.F.L. Ward, BU-HEPP-17-04, arXiv:1809.01492; BU- HEPP-17-05.

[21] B. Shakerin and B.F.L. Ward, BU-HEPP-18-02.

[22] B.F.L. Ward et al., PoS(RADCOR2017) (2018)

[23] S. Jadach, B.F.L. Ward, Z. A. Was and S. A. Yost, Phys. Rev. D 94 (2016) 074006.

[24] M. Aaboud et al., arXiv:1701.07240. 